\title{
10000 Two Cases of Cerebral Venous Sinus Technical Thrombosis Successfully Recanalized by the Concomitant Use of an Aspiration through the Guiding Catheter and Stent Thrombectomy Device
}

Toshiaki Bando, Yoji Kuramoto, Narihide Shinoda, Shinya Hori, Kazuyuki Mikami, Daisuke Shimo, Takahiro Kuroyama, and Yasushi Ueno

Objective: Two cases of cerebral venous sinus thrombosis (CVST) effectively recanalized endovascularly by a combination of thromboaspiration using Cerulean catheter DD6 and stent retriever device are reported.

Case Presentations: We endovascularly treated two cases of CVST that presented with progressive vomiting and disturbance of consciousness several days before the diagnosis of occlusion of the main venous sinus and venous congestion. The thrombus was removed by the combined use of an aspiration system made of Cerulean catheter DD6 connected to the Penumbra aspiration pump and a Solitaire FR thrombectomy device. In one case, balloon thrombofragmentation was performed with local thrombolytic therapy. In both cases, the occluded venous sinus was recanalized, venous congestion was resolved, and early initiation of anticoagulation therapy was possible. After the procedure, neurologic symptoms disappeared, and both patients were discharged 16 days after the procedure with a modified Rankin Scale (mRS) score of 0 .

Conclusion: In CVST, thromboaspiration by the concomitant use of a Cerulean catheter DD6 and a stent retriever is considered an effective therapeutic approach.

Keywords > cerebral venous sinus thrombosis, Penumbra aspiration pump, Cerulean catheter DD6, Solitaire FR, endovascular treatment

\section{Introduction}

In cerebral venous sinus thrombosis (CVST), the venous pressure increases due to occlusion of the venous sinus, and if the venous return is disturbed, the condition eventually progresses to venous infarction and venous hemorrhage, leading to a poor neurologic outcome. Therefore,

Department of Neurosurgery, Shinko Hospital, Kobe, Hyogo, Japan

Received: January 5, 2017; Accepted: July 21, 2017

Corresponding author: Toshiaki Bando. Department of Neurosurgery, Shinko Hospital, 1-4-47 Wakinohama-cho, Chuo-ku, Kobe, Hyogo 651-0072, Japan

Email: toshiakibando4@gmail.com

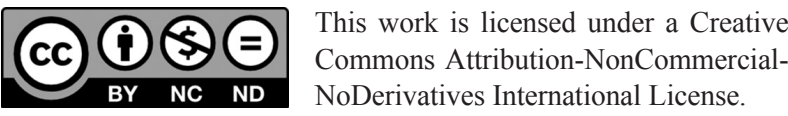

(C)2018 The Japanese Society for Neuroendovascular Therapy early recanalization of the major venous sinus is an important point in its treatment.

We report two cases of CSVT with occlusion of a major venous sinus and venous congestion presenting with progressive neurologic symptoms in which effective recanalization could be achieved by thromboaspiration concomitantly using a Cerulean DD6 (Medikit, Tokyo, Japan) connected to the Penumbra aspiration system (Penumbra, Alameda, CA, USA) and a stent retriever.

\section{Case Presentations}

\section{Case 1}

The patient was a 64-year-old male who had developed headache from 2 weeks before and vomiting from the day before the visit to the neurosurgery department of a local hospital and was referred to our hospital due to suspected CVST. He had histories of hypertension, dyslipidemia, and 


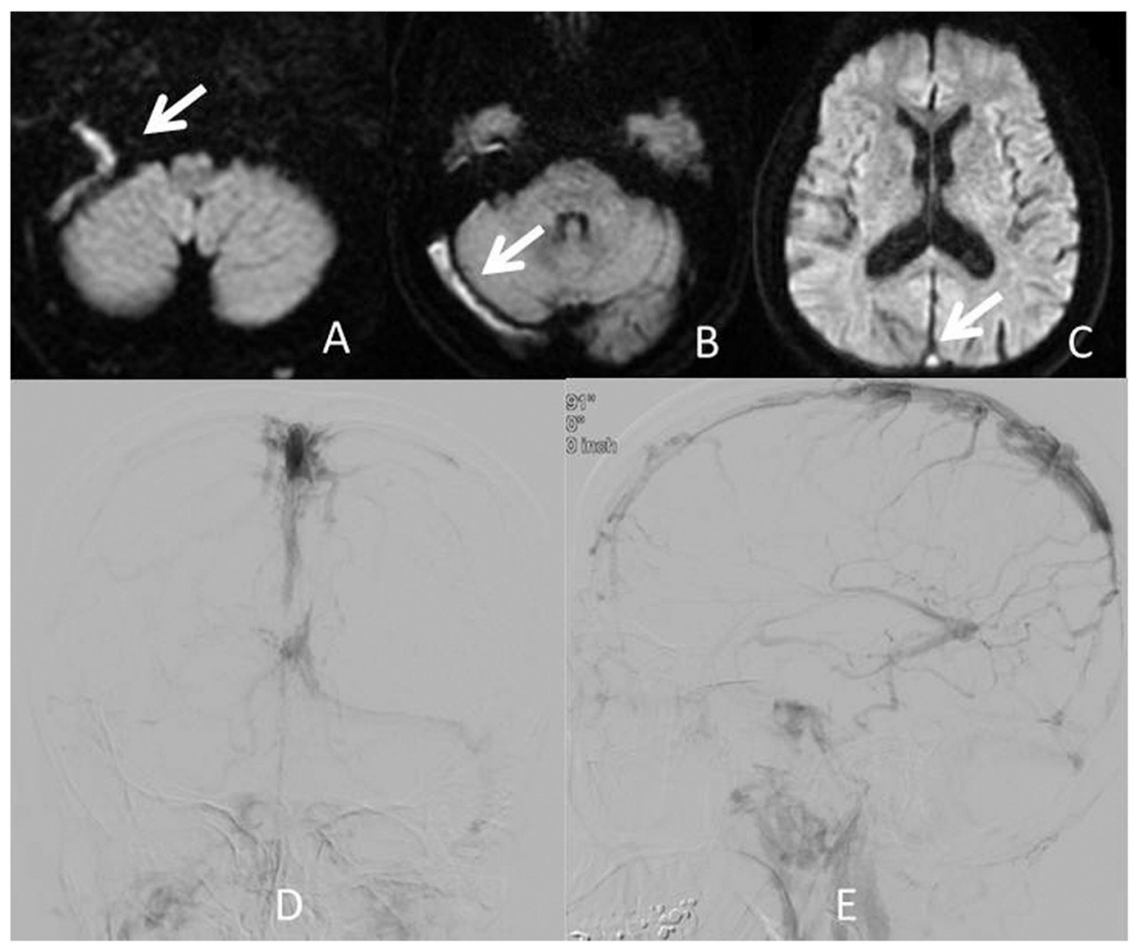

Fig. 1 (A-C) MR-DWI showed hyper-intensity object which was suspicious of the thrombus from SSS behind to right IJV (arrows). (D-E) Venous phase of right carotid angiogram showed complete occlusion from the SSS to right TS, and confluence was partially occluded. IJV: internal jugular vein; MR-DWI: magnetic resonance diffusion-weighted image; SSS: superior sagittal sinus; TS: transverse sinus

hyperuricemia. His medication history included amlodipine besilate, bezafibrate, and allopurinol.

On arrival, he showed a Japan Coma Scale (JCS) 0 state of consciousness, headache, and vomiting, but no clear neurologic deficits.

On blood tests, the cell counts, blood chemistry, clotting parameters, immunological indices, and tumor markers were all normal.

Concerning imaging findings, head MRI disclosed thrombosis from behind the superior sagittal sinus (SSS) predominantly to the right transverse sinus (TS)-sigmoid sinus (SS) and to the right internal jugular vein (IJV). Cerebral angiography (DSA) showed complete occlusion from behind the SSS to the right TS, partial occlusion of the confluence, reduced perfusion of the left TS and venous congestion (Fig. 1). No clear intracranial hemorrhage or subarachnoid hemorrhage was noted.

Since the patient had a 2-week history of progressive symptoms accompanied due to occlusion of the major venous sinus and marked venous congestion, endovascular treatment was selected for early resolution of symptoms and avoidance of hemorrhage due to congestion.
Heparin was intravenously injected at 4000 units. A 6 Fr. FUBUKI Dilator Kit STR $80 \mathrm{~cm}$ (ASAHI INTECC J-sales, INC., Nagoya, Japan) was placed in the right IJV, and the occluded right TS was recanalized with a 6 Fr. Cerulean DD6 STA $118 \mathrm{~cm}$ using a coaxial system consisting of a 4.2 Fr. FUBUKI $130 \mathrm{~cm}$ (ASAHI INTECC J-sales, INC.) and a Radifocus 0.035 inch $150 \mathrm{~cm}$. The Cerulean DD6 could be guided easily to a point near the thrombus behind the SSS using the 4.2 Fr. FUBUKI as an inner catheter. The 4.2 Fr. FUBUKI $130 \mathrm{~cm}$ was extracted from the Cerulean DD6, a Marksman (Medtronic, Minneapolis, MN, USA) was guided to a point distal to the thrombus using a Traxcess $14200 \mathrm{~cm}$ (Terumo Corporation, Tokyo, Japan), and a Solitaire FR $6 \times 30 \mathrm{~mm}$ (Medtronic, Dublin, Ireland) was deployed at the site of thrombosis. The 6 Fr. Cerulean DD6 was connected to the Penumbra aspiration pump, and a large volume of red thrombus could be retrieved by performing aspiration while withdrawing the entire system. Thrombectomy was performed three times at the site of occlusion by the same method, the SSS to the left TS could be recanalized, and the blood flow was improved. At this point, the right TS was unchanged, but 


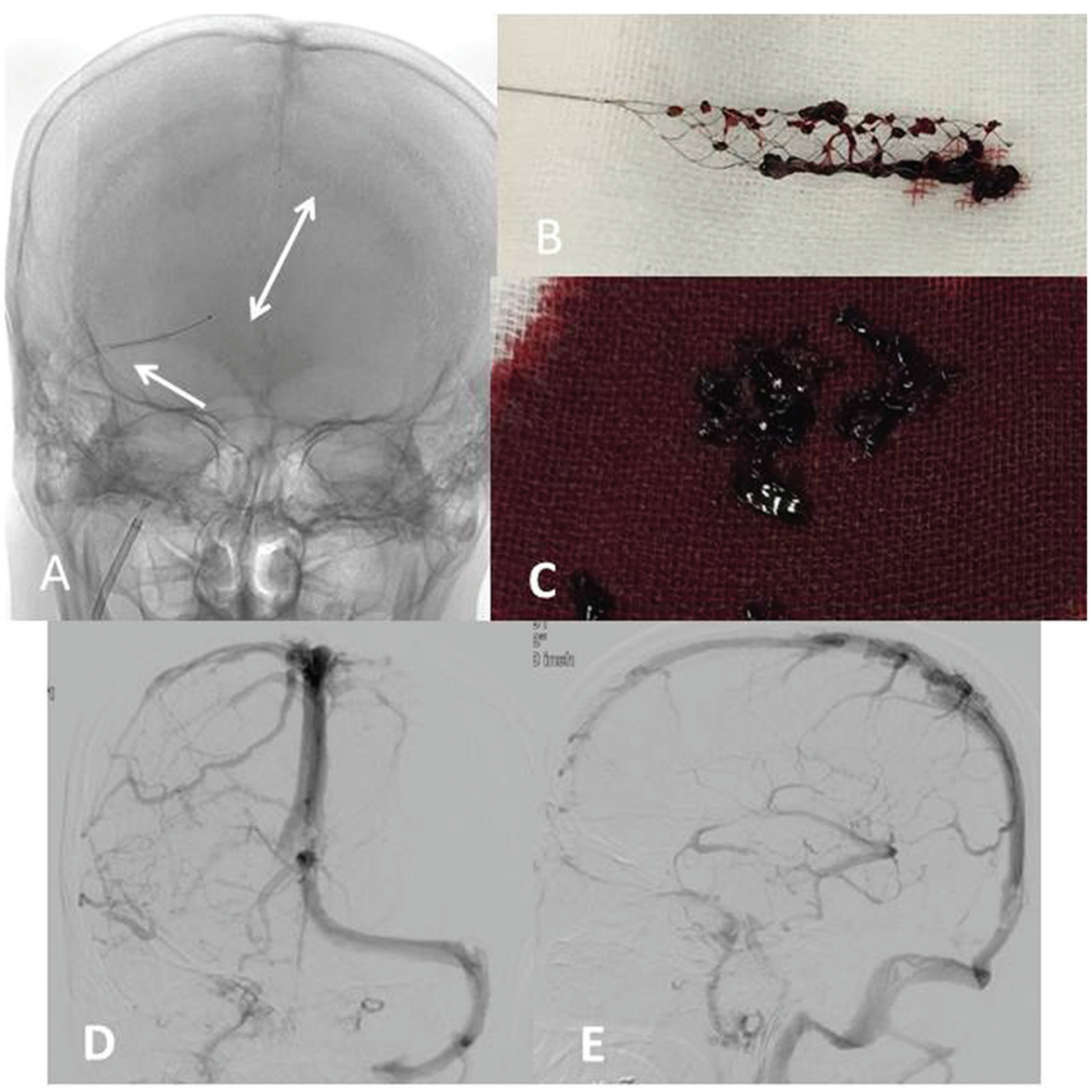

Fig. 2 (A) Solitaire-FR $6 \times 30 \mathrm{~mm}$ (double-headed arrow) was deployed and continuous aspiration from 6 Fr. Cerulean DD6 (Medtronic, Dublin, Ireland) (arrow). (B) Removed clots on Solitaire. (C) Removed clots from cerulean DD6. (D-E) Final DSA showed left TS was completely recanalized, and right TS was partially recanalized, also venous congestion was improved. TS: transverse sinus

partial recanalization was achieved by performing the same thrombectomy procedure once. Confirmation angiography showed improvement in perfusion from the SSS to the confluence and left TS with alleviation of left venous congestion (Fig. 2). While partial occlusion of the right TS persisted, we ended the treatment at this point by judging that the objective of treatment was achieved as the cerebral venous drainage tract could be secured with alleviation of left venous congestion.

Continuous intravenous infusion of heparin was performed after the procedure. Next day, the headache and vomiting were disappeared, so he took an oral warfarin instead of heparin infusion. No occurrence of neurologic deficits was observed. DSA was performed 7 days after the procedure showed patency of the recanalized sinuses, and MRI 52 days after the procedure confirmed the absence of thrombus behind the SSS to the right IJV (Fig. 3). The patient was discharged 16 days after the procedure with a modified Rankin Scale (mRS) score of 0.

\section{Case 2}

The patient was a 21-year-old male with no particular clinical history or medication history. He had developed progressive severe headache and disturbance of consciousness from 3 days before and was transported by ambulance. On arrival, the consciousness was JCS10-R, and severe headache and restlessness were noted, but no other local symptoms were evident.

On the blood tests, the Antithrombin III (ATIII) activity was $49 \%$, ATIII antigen was $11.6 \mathrm{mg} / \mathrm{dL}$, and $\alpha 2 \mathrm{MG}$ was $252 \mathrm{mg} / \mathrm{dL}$.

Head CT and head MRI showed thrombosis from behind the SSS to the bilateral TS, and MR susceptibility-weighted imaging (MR-SWI) of the head showed marked congestion of the cortical veins, primarily, of the right hemisphere. On DSA, the SSS was occluded, the right TS was completely occluded, and the left TS was partially occluded (Fig. 4). No clear intracranial hemorrhage or subarachnoid hemorrhage was noted. 


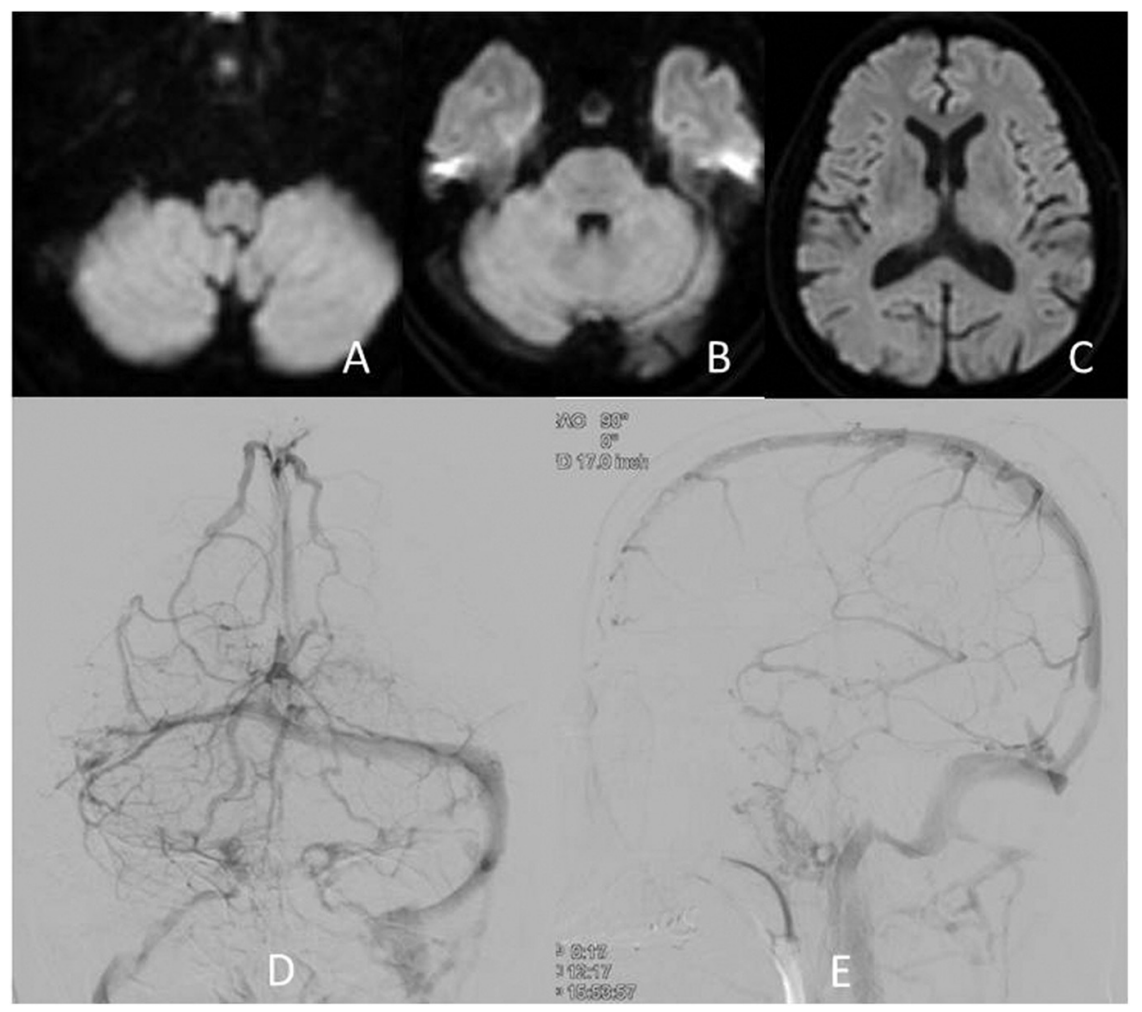

Fig. 3 (A-C) Follow-up on 52 days after treatment, MR-DWI showed disappearance of thrombus from SSS behind to right IJV. (D-E) Follow-up on 7 days after treatment, DSA showed improvement of anterograde sinus flow. IJV: internal jugular vein; MR-DWI: magnetic resonance diffusion-weighted image; SSS: superior sagittal sinus

Since the patient had progressive symptoms of 3-day duration, was difficult to sedate due to restlessness, and had occlusion of major venous sinuses accompanied by venous congestion, endovascular treatment was selected to alleviate symptoms and prevent exacerbation of the condition by early recanalization.

On DSA, the left IJV was clearly delineated, but the right IJV was obscure. While venous congestion of the right hemisphere suggested that it was optimal to approach the lesion via the right IJV, the left IJV was selected as the approach route in consideration of the safety of device navigation. Heparin was intravenously injected at 4000 units. An 8 Fr. Brite tip (Johnson \& Johnson, Fremont, CA, USA) was placed in the left IJV, and the occluded part of the SSS was passed using Excelsior SL-10 (Stryker, Kalamazoo, MI, USA) and CHIKAI $14200 \mathrm{~cm}$ (ASAHI INTECC $\mathrm{J}$-sales, INC.) in combination. While gradually descending the devices proximally from a point distal to the thrombus, UK was intravenously injected topically at $12 \times 10^{4}$ units. Next, balloon thrombofragmentation was performed a total of 16 times using a Jackal $4.0 \times 40 \mathrm{~mm}$ (Kaneka Medical Products, Osaka, Japan) and a Jackal $5.0 \times 40 \mathrm{~mm}$, but effective recanalization could not be obtained. A Solitaire FR $6 \times 30 \mathrm{~mm}$ was guided to a point distal to the thrombus using a coaxial system consisting of a 6 Fr. Cerulean DD6 STA $118 \mathrm{~cm}$, a Marksman, and a CHIKAI $14200 \mathrm{~cm}$ and was deployed at the site of thrombosis. The Cerulean DD6 was connected to the Penumbra aspiration pump, and, by performing aspiration while withdrawing the entire system, a large volume of red thrombus could be retrieved in the DD6 (Fig. 4). Thrombectomy was performed two times by the same procedure, resulting in persistence of delayed drainage of the venous sinus but partial recanalization from behind the SSS to the left TS and alleviation of congestion of the right cortical veins (Fig. 5). With alleviation of right venous congestion after recanalization of the SSS to the left TS, headache and restlessness were also resolved. While no change was observed in right TS occlusion, we judged that the objective of treatment was achieved and ended the treatment at this point.

Blood tests on admission showed a reduced ATIII activity, low ATIII antigen level, and high $\alpha 2 \mathrm{MG}$ level, and a diagnosis of CSVT due to ATIII deficiency was made. After endovascular treatment, an ATIII preparation was 


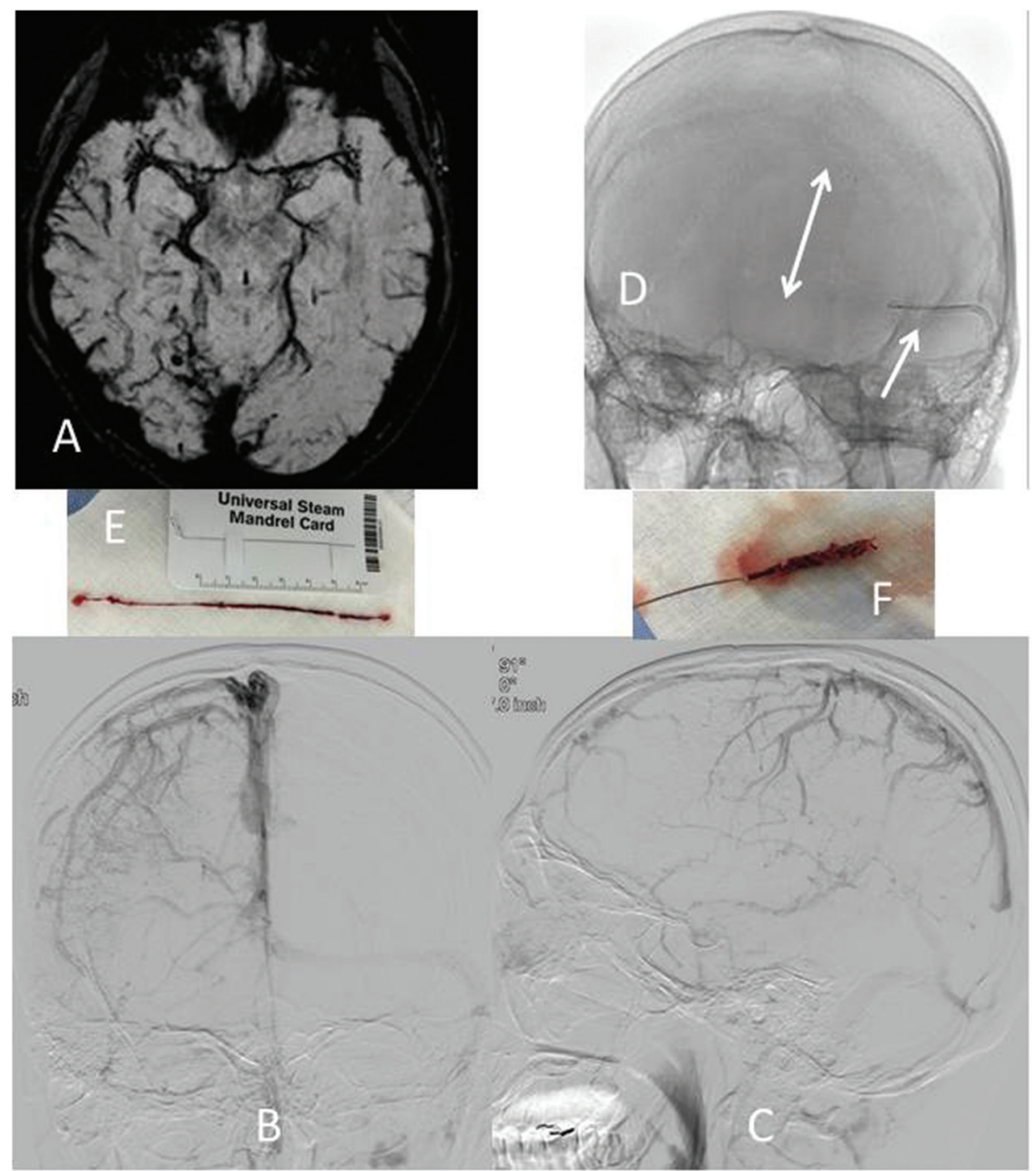

Fig. 4 (A) MR-SWI showing marked venous congestion on right occipital lobe. (B-C) DSA showing total occlusion of SSS and right TS, partial occlusion of left TS. (D) Solitaire-FR $6 \times$ $30 \mathrm{~mm}$ (Medtronic, Dublin, Ireland) (double-headed arrow) was deployed and continuous aspiration from 6 Fr. Cerulean DD6 (Medikit, Tokyo, Japan) (arrow). (E) Removed clots from cerulean DD6. (F) Removed clots on Solitaire-FR. MR-SWI: magnetic resonance susceptibility weighted imaging; SSS: superior sagittal sinus; TS: transverse sinus

administered simultaneously with continuous intravenous infusion of heparin, and the regimen was changed thereafter to an oral warfarin. DSA performed 12 days after the procedure showed improved delineation of the right TS (Fig. 5). The patient was discharged 16 days after the procedure with a mRS score of 0 .

\section{Discussion}

CVST affects relatively young individuals, but its incidence is low, accounting for $0.5 \%-1.0 \%$ of all strokes. It is caused by congenital (anticoagulation protein deficiency) or acquired factors (trauma, infection, pregnancy, antiphospholipid antibody syndrome, malignant tumor, oral contraceptives, etc.). It exhibits a variety of symptoms including headache, convulsion, nausea/vomiting, disturbance of consciousness, hemiplegia, and aphasia, and the SSS and TS account for $70 \%-90 \%$ of the occluded venous sinuses. Occlusion of venous sinuses increases the venous pressure, and if it also disturbs the venous perfusion, the condition progresses eventually to venous infarction and venous hemorrhage with a poor neurologic outcome. Therefore, early recanalization of the occluded major venous sinuses is an important point in its treatment. ${ }^{1)}$ Anticoagulant therapy is the first choice, ${ }^{2}$ but if CVST is resistant to usual anticoagulant therapy, or if prompt resolution of symptoms associated with venous congestion such as severe nausea and disturbance of consciousness is necessary, endovascular treatment is indicated. A randomized controlled trial (RCT) to compare the effectiveness of 


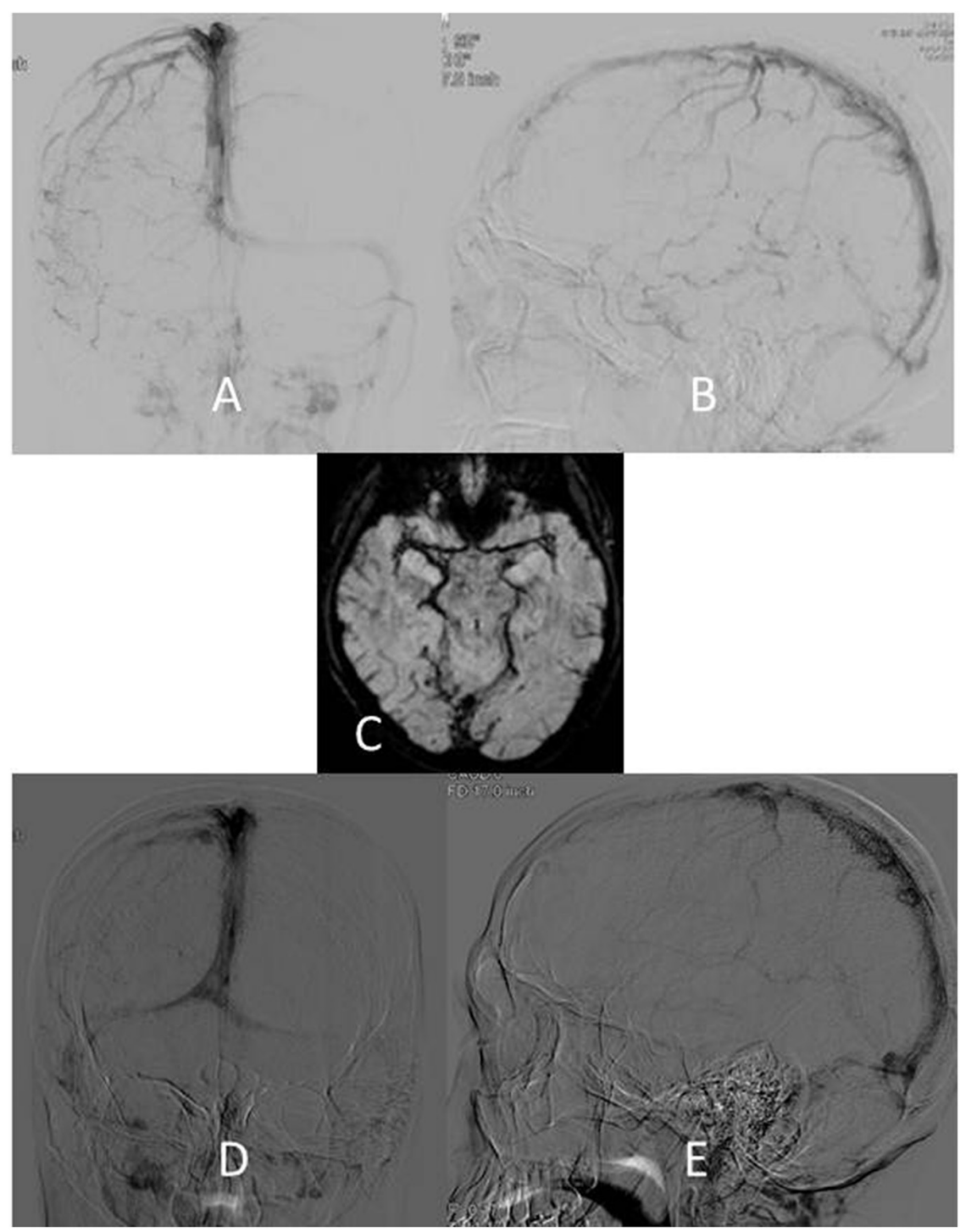

Fig. 5 (A and B) Final DSA showed partial recanalization of SSS and left TS, also right cerebral venous congestion was improved. (C) Follow-up on 11 days after treatment, MR-SWI showed improvement of right occipital venous congestion. (D-E) Follow-up on 12 days after treatment, DSA showed improvement of anterograde sinus flow from SSS to right TS. MR-SWI: magnetic resonance susceptibility weighted imaging; SSS: superior sagittal sinus; TS: transverse sinus

endovascular treatment with that of heparin anticoagulant therapy is in progress, and its results are awaited. ${ }^{3)} \mathrm{We}$ selected endovascular treatment in the two patients reported here because they showed progressive headache, nausea, and restlessness and were judged to need prompt control of symptoms. This resulted in early recovery of venous perfusion and discharge of both patients 16 days after the procedure without symptoms.

Endovascular treatments for CVST include thrombolytic therapy in the venous sinus using recombinant tissuetype plasminogen activator (rt-PA) or urokinase, AngioJet Rheolytic catheter, balloon angioplasty, stent placement, mechanical thrombofragmentation using devices such as the microsnare, mechanical thrombectomy using a stent retriever, and thromboaspiration using the Penumbra aspiration system. ${ }^{4-7)}$ Methods such as thromboaspiration combined with conventional thrombolytic therapy, mechanical thrombofragmentation, and mechanical thrombectomy have also been reported. ${ }^{8-15)}$ According to the systematic review of endovascular treatment for CVST performed at 42 institutions (185 cases) between January 1995 and February 2014 by Siddiqui et al., the mainstay of treatment was AngioJet (40\%), followed by the snare (31\%), balloon angioplasty (25\%), and Penumbra system (7\%), and 


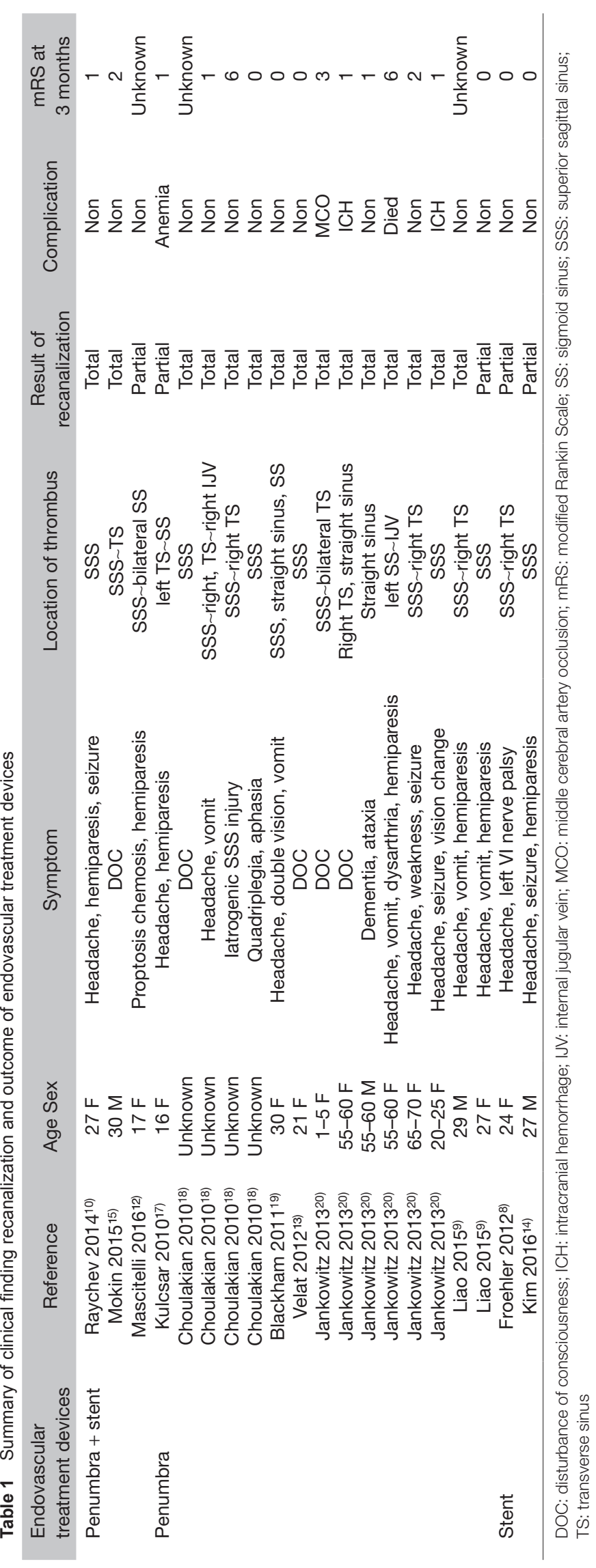


thrombolytic therapy was performed in $71 \%$ of the patients. However, the complete recanalization rate by AngioJet was only $55 \%$, and this low recanalization rate was associated with a poor outcome and complications. ${ }^{16)}$ The recanalization rate is considered to be higher by mechanical thrombectomy and thromboaspiration than by mechanical thrombofragmentation or thrombolytic therapy because a large volume of thrombus can be retrieved at one time, and treatments by the Penumbra aspiration system alone, stent retriever alone, and a combination of the Penumbra and stent retriever have been reported to be effective (Table 1). This combination is considered to improve the recanalization rate since a large volume of thrombus can be retrieved at one time including thrombus at the origins of cortical veins as well as that in major venous sinuses. ${ }^{6}$ )

In this study, we selected a combination of the Penumbra aspiration system and a stent retriever as endovascular therapy for CVST. In addition, as a modification, the Cerulean DD6 was used by connecting it to the Penumbra aspiration system. The 6 Fr. Cerulean DD6 (internal diameter: 0.072 inch) has a lumen larger than that of Penumbra 5MAX ACE (internal diameter: 0.060 inch) and a greater thrombus retrieving ability and was considered to be more useful for the treatment of CVST, in which a large volume of thrombus must be removed. ${ }^{16)}$

Moreover, we also modified two points: safe navigation of the Cerulean DD6 in the venous sinus and thrombus retrieval. Concerning the first point, navigation of the Cerulean DD6 using a microcatheter and a microguidewire was difficult in Case 1, but it was facilitated by the use of a 4.2 Fr. FUBUKI and a Radifocus 0.035 inch. However, compared with the microguidewire, the Radifocus 0.035 inch has a greater risk of vascular perforation if it erroneously enters a bridging vein. Therefore, sufficient fluoroscopic confirmation of its position is necessary. The second point is simultaneously performing stent retrieval and pump aspiration. According to the literature, Marksman was reported to be completely withdrawn from the aspiration catheter during pump aspiration to enhance the aspiration efficiency of venous thrombus, ${ }^{6}$ ) but we kept Marksman and made pump aspiration while holding the venous thrombus with the stent and thus could retrieve a large volume of venous thrombus at one time without omission. In addition, by sufficiently washing the entire system including the Cerulean DD6 with heparinized saline after each recovery of the stent retriever, thrombus formation and attachment of thrombus in the catheter could be prevented.
The method that we have proposed has limitations. While no luminal collapse is observed by usual aspiration using the Penumbra aspiration system within the limit of aspiration pressure $(-67.7 \mathrm{kPa})$, there is a possibility of its occurrence when the Cerulean DD6 is used because the limit of aspiration pressure with Cerulean DD6 is unknown. In actual treatment, however, no luminal collapse was noted even when aspiration was performed by connecting the Cerulean DD6 to the pump, and it could be used similarly to the Penumbra aspiration system. Also, since the Cerulean DD6 is less flexible than the Penumbra, whether or not it can be applied to all cases of CVST is unclear. Further accumulation of cases and validation are necessary concerning these points for the future.

\section{Conclusion}

In endovascular treatment for CVST, thromboaspiration with a combination of the Cerulean DD6 connected to the Penumbra aspiration system and a stent retriever is considered a safe and effective procedure that is expected to yield a high recanalization rate.

\section{Disclosure Statement}

The first author and the coauthors have no conflicts of interest.

\section{References}

1) Saposnik G, Barinagarrementeria F, Brown RD, et al: Diagnosis and management of cerebral venous thrombosis: a statement for healthcare professionals from the American Heart Association/American Stroke Association. Stroke 2011; 42: 1158-1192.

2) Ferro JM, Crassard I, Coutinho JM, et al: Decompressive surgery in cerebrovenous thrombosis: a multicenter registry and a systematic review of individual patient data. Stroke 2011; 42: 2825-2831.

3) Coutinho JM, Ferro JM, Zuurbier SM, et al: Thrombolysis or anticoagulation for cerebral venous thrombosis: rationale and design of the TO-ACT trial. Int J Stroke 2013; 8: 135-140.

4) Baker MD, Opatowsky MJ, Wilson JA, et al: Rheolytic catheter and thrombolysis of dural venous sinus thrombosis: a case series. Neurosurgery 2001; 48: 487-493; discussion 493-494.

5) Curtin KR, Shaibani A, Resnick SA, et al: Rheolytic catheter thrombectomy, balloon angioplasty, and direct 
recombinant tissue plasminogen activator thrombolysis of dural sinus thrombosis with preexisting hemorrhagic infarctions. AJNR Am J Neuroradiol 2004; 25: 1807-1811.

6) Khan SH, Adeoye O, Abruzzo TA, et al: Intracranial dural sinus thrombosis: novel use of a mechanical thrombectomy catheter and review of management strategies. Clin Med Res 2009; 7: 157-165.

7) Adachi H, Mineharu Y, Ishikawa T, et al: Stenting for acute cerebral venous sinus thrombosis in the superior sagittal sinus. Interv Neuroradiol 2015; 21: 719-723.

8) Froehler MT: Successful treatment of cerebral venous sinus thrombosis with the Solitaire FR thrombectomy device. J Neurointerv Surg 2013; 5: e45. doi: 10.1136/neurintsurg-2012-010517.

9) Liao W, Liu Y, Gu W, et al: Cerebral venous sinus thrombosis: successful treatment of two patients using the penumbra system and review of endovascular approaches. Neuroradiol J 2015; 28: 177-183.

10) Raychev R, Tateshima S, Rastogi S, et al: Successful treatment of extensive cerebral venous sinus thrombosis using a combined approach with Penumbra aspiration system and Solitaire FR retrieval device. J Neurointerv Surg 2014; 6: e32.

11) Shaikh H, Pukenas BA, McIntosh A, et al: Combined use of Solitaire FR and Penumbra devices for endovascular treatment of cerebral venous sinus thrombosis in a child. J Neurointerv Surg 2015; 7: e10.

12) Mascitelli JR, Pain M, Zarzour HK, et al: Sinus thrombectomy for purulent cerebral venous sinus thrombosis utilizing a novel combination of the Trevo stent retriever and the Penumbra ACE aspiration catheter: the stent anchor with mobile aspiration technique. J Neurointerv Surg 2016; 8: e24. doi: 10.1136/neurintsurg-2015-011782.

13) Velat GJ, Skowlund CJ, Waters MF, et al: Direct thrombectomy using the Penumbra thromboaspiration catheter for the treatment of cerebral venous sinus thrombosis. World Neurosurg 2012; 77: 591.e15- 591.e18.

14) Kim H, Kim SR, Park IS, et al: Treatment of the superior sagittal sinus thrombosis with the mechanical thrombectomy using stent-retriever device. J Korean Neurosurg Soc 2016; 59: 518-520.

15) Mokin M, Lopes DK, Binning MJ, et al: Endovascular treatment of cerebral venous thrombosis: contemporary multicenter experience. Interv Neuroradiol 2015; 21: 520-526.

16) Siddiqui FM, Dandapat $S$, Banerjee $C$, et al: Mechanical thrombectomy in cerebral venous thrombosis: systematic review of 185 cases. Stroke 2015; 46: 1263-1268.

17) Kulcsár Z, Marosfoi M, Berentei Z, et al: Continuous thrombolysis and repeated thrombectomy with the Penumbra System in a child with hemorrhagic sinus thrombosis: technical note. Acta Neurochir (Wien) 2010; 152: 911-916.

18) Choulakian A, Alexander MJ: Mechanical thrombectomy with the penumbra system for treatment of venous sinus thrombosis. J Neurointerv Surg 2010; 2: 153-156.

19) Blackham KA: Extensive dural sinus thrombosis: successful recanalization with thrombolysis and a novel thrombectomy device. J Neurosurg 2011; 114: 133-135.

20) Jankowitz BT, Bodily LM, Jumaa M, et al: Manual aspiration thrombectomy for cerebral venous sinus thrombosis. J Neurointerv Surg 2013; 5: 534-538. 